\title{
Physical Examination Sponsor Defined Identifier
}

National Cancer Institute

\section{Source}

National Cancer Institute. Physical Examination Sponsor Defined Identifier. NCI

Thesaurus. Code C83080.

One or more sponsor-defined characters used to identify, name, or characterize the physical examination. 